\title{
Twins twin transfusion syndrome in monochorionic twins
}

\begin{abstract}
Objective: To report our experience with the Monochorionic Diamniotic pregnancies complicated by Twin-Twin Transfusion Syndrome (TTTS) in a large retrospective cohort study. It emphasizes on the clinical prognostic implication of TTTS, the importance of timely surveillance and intervention in an attempt to prevent the increased fetal/perinatal morbidity and mortality.
\end{abstract}

Materials and Methods: his retrospective study was conducted at Women's Hospital, Hamad Medical Corporation, Qatar (2003-Oct.2014) including all the Monochorionic Diamniotic twins who were complicated by TTTS. The data included the maternal biodata, method of conception and maternal complications, ultrasound findings and follow-ups, intervention (if any), delivery gestational age was as well as the birth/miscarriage data, and birth weight. We kept these data anonymously in a password protected Excel sheet and the analysis carried using online statistics tools.

Results: 18 cases of TTTs diagnosed among 206 Monochorionic diamniotic twin $(8.7 \%)$, The mean gestational age at diagnosis is $23 \pm 4$ weeks. $39 \%$ had an advanced TTTS (stage 3 , 4 and 5). Mean maternal age was $29.5 \pm 4.9$ years, versus $30.2 \pm 5.7$ years of No-TTTS cases (p-value 0.6 ). The rate of miscarriage was $28 \%$ and IUFD was $27 \%$, the mean diagnosisIUFD interval was $1.6 \pm 1.6$ weeks. The mean gestation age at delivery was $27.2 \pm 4.6$ weeks versus $30.2 \pm 5.7$ weeks for the Non-TTTS (p-value 0.0001 ). The mean birth weight for the alive recipients after 24 weeks was $1364 \pm 564$ grams versus $907.6 \pm 289.9$ for the donor (p-value 0.04)

Conclusion: Our research highlights the continuous devastating complications of monochorionic twins. It is our duty as health professionals to provide a comprehensive counselling as early as chorionicity regarding these risks and their implications on the mother and fetus/neonate.
Volume 5 Issue 5 - 2019

\begin{abstract}
Mohamed W Gawish, AAAI Ibrahim, Sawsan Al Obaidly, Mohamed Ali Mustafa Khalil, Mohamed Ahmed Abdelmoneam Ramadan Department of Obstetrics and Gynaecology, Hamad Medical Corporation, Qatar
\end{abstract}

Correspondence: MW Gawish, Department of Obstetrics and Gynaecology,Women's Hospital, Hamad Medical Corporation, Doha, Qatar, Email dr.m.gawish1984@gmail.com

Received: September 15, 2019 | Published: October 04, 2019

\section{Introduction}

Twin-twin transfusion syndrome (TTTS) is a disease that is derived from the unbalanced sharing of blood between fetuses via placental anastomosing vessels. Technically, the disease occurs between two or more fetuses. As such, the proper term to refer to the condition should be "fetofetal transfusion. ${ }^{1}$ It is a fascinating condition in which fetuses of identical genotype adopt discordant cardiovascular phenotypes, secondary to unbalanced placental inter-twin transfusion. Flow along the primary units of intertwin transfusion, unidirectional arteriovenous anastomoses, can be as high as liters/day each, and TTTS develops when the placenta has insufficient compensatory counter-transfusional anastomosis. ${ }^{2}$ In this research, we share our experience of this complicated condition, the management provided and the outcomes.

\section{Objective}

The aim of this research was to report our experience with the Monochorionic Diamniotic pregnancies and their antenatal complication of Twin Twin Transfusion Syndrome (TTTS) in a large retrospective cohort study. This research will emphasize on the clinical prognostic implication of Monochorionic Diamniotic twining and the increasing risk of antenatal complications among these pregnancies looking at the prevalence of TTTS, and the outcome of the both twins in attempt to introduce more active intervention in utero and antenatal management modalities to improve such result. The rationale for fetal surveillance in Monochorionic Diamniotic twin pregnancies is timely intervention to prevent the increased fetal/perinatal morbidity and mortality.

\section{Materials and methods}

This retrospective study was conducted at Women's Hospital, Hamad Medical Corporation, Qatar (2003-Oct.2014). All the Monochorionic Diamniotic twins cases reviewed in the study were seen in the Feto-Maternal Unit which is a specialized unit in the Obstetrics and Gynecology department which started on 2003 and serves high-risk pregnancies such as twin pregnancies.

The ultrasound scan data, particularly concerning chorionicity, and diagnosis of TTTs were obtained from the ultrasound software (Astraia Software GmbH Occamstr.20, 80802 Munich Germany) and the medical records for the mothers. The mother antenatal record including age, parity, race, method of conception and maternal complications such as preeclampsia and diabetes, were collected. All cases of fetal death in Monochorionic Diamniotic twins regardless of gestational age, are included in the study to report the outcome of the co-twins. The approximate time interval between the diagnosis of TTTS and delivery gestational age was calculated from information 
recorded in the case notes. The birth/miscarriage data, birth weight, were retrieved. The Data were kept in a password protected Excel sheet (C) 2010 Microsoft Corporation) and the analysis carried using online statistics tools (http://www.alcula.com/calculators/statistics/ dispersion/, http://www.socscistatistics.com/tests/). It was part of a clinical audit, so no ethical approval was required.

\section{Results}

18 cases of TTTs diagnosed among 206 Monochorionic diamniotic twin $(8.7 \%)$, The mean gestational age at diagnosis is $23 \pm 4$ weeks, the median of 23 weeks (Figure 1). Out of these cases, 7 cases of advanced TTTS [39\% ( 4 for stage 3, 2 for stage 4 and one for stage 5)]. However, there was no statistical difference between the proportion of those with advanced disease and those with earlystage (stage 1 and two, which encompass 62\%), p-value 0.15 . Mean maternal age was $29.5 \pm 4.9$ years, compared to non-TTTS cases where the mean is $30.2 \pm 5.7$ years, there was no statistical difference ( $p$-value $0.6,95 \%$ CI -2.04 to 3.4 ). Mean Parity was $1.4 \pm 1.6$. However, that did not significantly influence the development of TTTS compared to no-TTTS (p-value of 0.36). Among these cases, there was an eventual 5 IUFD-27\% (1 include both babies); the mean diagnosis-IUFD interval was $1.6 \pm 1.6$ weeks. For the four where the co-twin lived, 1 had hydrocephalus and eventual cerebral palsy. Five cases had a miscarriage before 24 weeks.

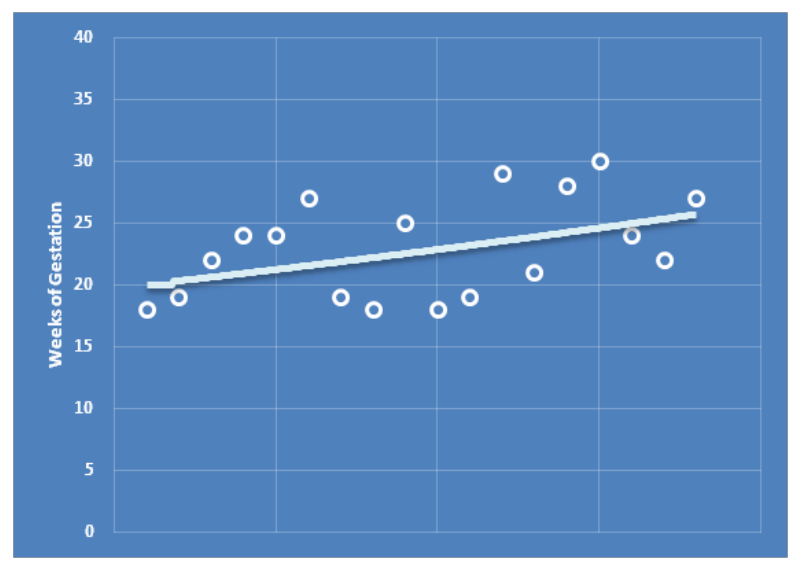

Figure I Gestational Age Distribution at Diagnosis of the TTTS cases.

The mean gestation age at delivery was $27.2 \pm 4.6$; the average diagnosis delivery interval was $4.2 \pm 4.7$ weeks. We noticed that there was a significant statistical difference between the diagnosis and the delivery (p-value 0.006) even with the 5 IUFDs. Mean gestational age for Non-TTTS was $30.2 \pm 5.7$ weeks, there was a significant difference between the two groups (p-value 0.0001) (Table 1). The mean birth weight for the alive recipients after 24 weeks was $1364 \pm 564$ weeks while the average birth weight of the donor was $907.6 \pm 289.9$ weeks, and there was a statically significant difference (p-value 0.04) (Figure 2).

Table I Patients Bio data

\begin{tabular}{|c|c|}
\hline Variable & Statistics \\
\hline Maternal Age (years) (Mean $\pm S D$ ) & $29.5 \pm 4.9$ \\
\hline Parity (Median) & 2 \\
\hline Mode of Conception Spontaneous & 17 (94.4\%) \\
\hline
\end{tabular}

\begin{tabular}{lll}
$\begin{array}{ll}\text { Table continued } \\
\text { Variable }\end{array}$ & Statistics \\
\hline \multirow{3}{*}{ Medical disease } & IVF & I $(5.6 \%)$ \\
& Gypothyroid & I $(5.6 \%)$ \\
Gestational age at Dx (Weeks) (Mean \pm 4.9$)$ & $4(27.8 \%)$ \\
Rate of IUFD of One Twin & $23 \pm 4$ \\
Rate of Miscarriage & & $28 \%$ \\
Birth weight & $\begin{array}{l}\text { Recipient (grams) } \\
\text { (Mean } \pm \text { SD) }\end{array}$ & $1364 \pm 564$ \\
& $\begin{array}{l}\text { Donor (grams) } \\
\text { (Mean } \pm \text { SD) }\end{array}$ & $907.6 \pm 289.9$ \\
\hline
\end{tabular}

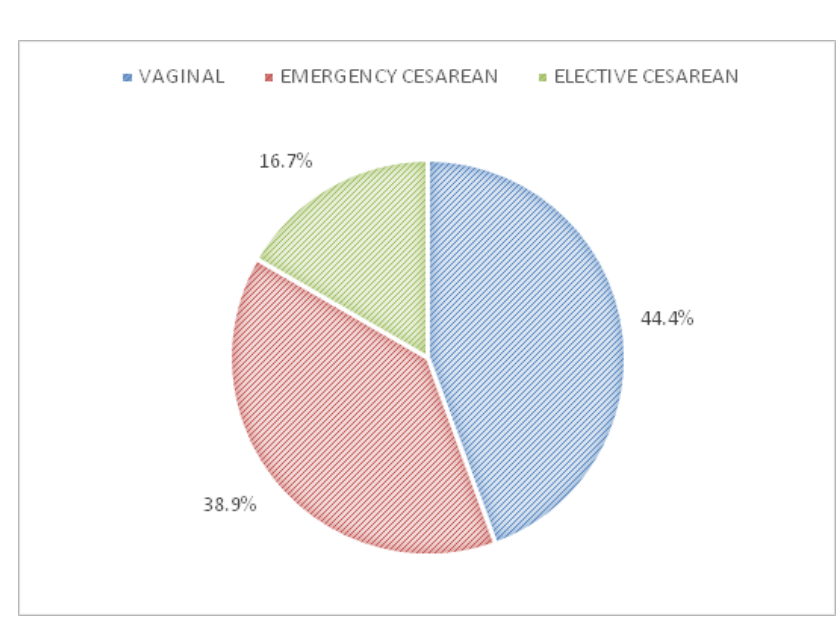

Figure 2 Mode of delivery.

\section{Discussion}

Twin-twin transfusion syndrome (TTTS) is a serious complication of Monochorionic twins. Untreated, it results in inordinate perinatal mortality and morbidity. ${ }^{3}$ This study shows how devastating Monochorionic pregnancy can be if complicated by TTTS. Twintwin transfusion syndrome (TTTS) is a severe complication affecting approximately $15 \%$ of Monochorionic (MC) twin pregnancies as a result of unbalanced placental vascular anastomosis. ${ }^{4}$ In our study the rate was $8.7 \%$, this could be attributed to the fact that fact that many cases abort or end up with IUFD before referral to the Specialized Fetal-maternal Unit, the other factor is misdiagnosis which is attributed to many factors include: poor recognition of chorionicity in early pregnancy (11-14 weeks); suboptimal knowledge amongst health-care providers and patients regarding $\mathrm{MC}$ twin complications; inadequate ultrasound follow-up of $\mathrm{MC}$ pregnancies; failure to recognize the urgency of TTTS, with subsequent delay in referral. ${ }^{5}$

Quintero staging system defines five stages of TTTS. In stage I, there is polyhydramnios in the recipient and oligohydramnios in the donor, with the bladder of the donor twin remaining visible. Umbilical Doppler studies are not critically abnormal in stage I. Stage II is categorized by continuing polyhydramnios/oligohydramnios, but with no urine visible in the donor's bladder. The donor is termed stuck because the amniotic sac adheres to the fetus, leaving minimal or no room for movement. Stage III begins when conflicting amniotic fluid levels are complicated by abnormal cord Doppler studies. In stage 
IV, there is an occurrence of hydrops or fluid gathering in two or more cavities of the donor or recipient. It is important to note that the existence of hydrops is a poor predictive sign. If the syndrome advances to stage $\mathrm{V}$, fetal demise can occur in either fetus. ${ }^{6}$ In our Study there were heterogeneous presentations encompassing all deferent stages, one of the reasons to that is the late referral (see misdiagnosis factors above) and the lack of definitive treatment in the country (i.e., laser therapy).

IUFD is one of the complications of untreated TTTS, in our series the rate was $27 \%$ rate of IUFD, added to 3 cases of mid-trimester miscarriage (total of $38 \%$ ) which not comparable to the literature of up to $80 \%$, the reason for that is the iatrogenic prematurity that's conducted after 28 weeks in those cases and of course the limited number of cases. All donor twins who delivered were growth restricted compared to a recipient, which could be (but not a must) part of the pathology. Preterm delivery is evident in our series, compared to uncomplicated cases, which attributed to two factors; the iatrogenic preterm deliveries mainly after 28 weeks; mostly by cesarean section (which add to the complications of TTTS), and the preterm delivery as a result of the diseases itself (severe polyhydramnios). ${ }^{7}$

\section{Conclusion}

Our research highlights the continuous devastating complications of monochorionic twins. It is our duty as health professionals to provide a comprehensive counselling as early as chorionicity regarding these risks and their implications on the mother and fetus/neonate. It is of utmost importance to diagnose the chorionicity as early as possible and refer them to a high-risk institute for further care.

\section{Acknowledgments}

None.

\section{Conflicts of interest}

All authors declare no conflict of interest.

\section{Funding details}

None.

\section{References}

1. Kontopoulos E, Chmait R, Quintero R. Twin-to-Twin Transfusion Syndrome: Definition, Staging, and Ultrasound Assessment. Twin Res Hum Genet. 2016;19(3):175-183.

2. Fisk N, Duncombe G, Sullivan M. The Basic and Clinical Science of Twin-Twin Transfusion Syndrome. Placenta. 2009;30(5):379-390.

3. Fisk, Nicholas M, Paula Galea. Twin-Twin Transfusion - As Good as It Gets? New England Journal of Medicine. 2004;351(2):182-184.

4. Mosquera C, Miller RS, Simpson LL. Twin-twin transfusion syndrome. Semin Perinatol. 2012;(36):182-189.

5. Baud D, Windrim R, Van Mieghem T, et al. Twin-twin transfusion syndrome: a frequently missed diagnosis with important consequences. Ultrasound Obstet Gynecol. 2014;(44):205-209.

6. Jackson KM, Mele NL. Twin-to-Twin Transfusion Syndrome: What Nurses Need to Know. Nursing for Women's Health. 2009;13(3):224 233

7. Smith RP, Denbow ML. Twin-to-twin transfusion syndrome. The Obstetrician \& Gynaecologist. 2006;8:1-6. 